\title{
PRESSURE-DEPENDENT EPANET EXTENSION
}

\author{
Calvin Siew ${ }^{1}$ and Tiku T. Tanyimboh ${ }^{2}$
}

Department of Civil Engineering, University of Strathclyde Glasgow. John Anderson Building, 107 Rottenrow, Glasgow G4 0NG, UK.

${ }^{1}$ PhD student, Department of Civil Engineering, University of Strathclyde Glasgow. John Anderson Building, 107 Rottenrow Glasgow G4 0NG, UK.

Email : calvin.siew@strath.ac.uk

Tel : +44(0) 1415483578

${ }^{2}$ Corresponding Author.

Senior Lecturer, Department of Civil Engineering, University of Strathclyde Glasgow. John Anderson Building, 107 Rottenrow Glasgow G4 0NG, UK.

Email : tiku.tanyimboh@strath.ac.uk

Tel : +44 (0)1415484366

FAX : +44 (0)1415532066

This article was published in Water Resources Management (2012) 26:1477-1498.

Water Resources Management

April 2012, Volume 26, Issue 6, pp 1477-1498

Pressure-Dependent EPANET Extension

Calvin Siew, Tiku T. Tanyimboh

The final publication is available at http://link.springer.com/article/10.1007/s11269011-9968-x.springer.com. 


\title{
PRESSURE-DEPENDENT EPANET EXTENSION
}

\author{
Calvin Siew and Tiku T. Tanyimboh \\ Department of Civil Engineering, University of Strathclyde Glasgow, UK
}

\begin{abstract}
In water distribution systems (WDSs), the available flow at a demand node is dependent on the pressure at that node. When a network is lacking in pressure, not all consumer demands will be met in full. In this context, the assumption that all demands are fully satisfied regardless of the pressure in the system becomes unreasonable and represents the main limitation of the conventional demand driven analysis (DDA) approach to WDS modelling. A realistic depiction of the network performance can only be attained by considering demands to be pressure dependent. This paper presents an extension of the renowned DDA based hydraulic simulator EPANET 2 to incorporate pressure-dependent demands. This extension is termed "EPANET-PDX" (pressure-dependent extension) herein. The utilization of a continuous nodal pressure-flow function coupled with a line search and backtracking procedure greatly enhance the algorithm's convergence rate and robustness. Simulations of real life networks consisting of multiple sources, pipes, valves and pumps were successfully executed and results are presented herein. Excellent modelling performance was achieved for analysing both normal and pressure deficient conditions of the WDSs. Detailed computational efficiency results of EPANET-PDX with reference to EPANET 2 are included as well.
\end{abstract}

Keywords Demand-driven analysis - Head-dependent modelling • Head-flow relationship - Pressure-dependent demand - Pressure-deficient water distribution system 


\section{Introduction}

Pressure deficient conditions are inevitable in water distribution systems (WDSs) and can be caused by common occurrences such as pump failure, pipe bursts, isolation of major pipes from the system for planned maintenance work (Park et al. 2010, Christodoulou 2010) and excessive fire fighting demands. Under these circumstances, the WDS may not be able to satisfy all consumer demands. This requires water companies to accurately model and analyse the WDS for crucial decision making. However, the widely used demand driven analysis (DDA) is inappropriate for modelling pressure deficient networks. This conventional model is formulated under the assumption that demands are fully satisfied regardless of the pressure and yields lower or even negative nodal pressure while analysing a pressure deficient network. Hence, DDA is unable to accurately quantify the exact magnitude of deficiency in terms of nodal pressure and outflow. This is critical information that cannot be overlooked during a WDS performance evaluation. The need for an analysis methodology that explicitly takes into account the relationship between nodal flows and pressure cannot be further stressed. This method is known as pressure dependent analysis (PDA) and models the WDS in a more realistic manner (Tabesh et al. 2009, Martinez-Rodriguez et al. 2011).

There are numerous methods of obtaining the available nodal flow for PDA in the literature. These methods can generally be categorized into two. The first category comprises of methods involving demand driven analysis. For example, Ang and Jowitt (2006) proposed an algorithm which progressively adds artificial reservoirs to pressure deficient nodes. The approach used is similar to that of Bhave (1991). Rossman (2007) implemented pressure dependent demands by modelling emitters as orifices at nodes. Kalungi and Tanyimboh (2003) developed a heuristic in which some aspects of PDA were used in a DDA environment to identify zero and partial flow nodes. Gupta and Bhave (1996) developed an iterative approach which adjusted nodal flows using several DDA runs. Aside from Rossman (2007), all of the methods mentioned involve repetitive use of DDA with successive adjustments made to specific parameters until a sufficient hydraulic consistency is obtained. This can lead to high computational requirement and may present difficulties to be effectively implemented for large networks.

The second category involves the approach where a head-flow relationship (HFR) is embedded in the system of hydraulic equations. HFRs are functions used to estimate the actual flow at demand nodes based on the nodal pressure. HFRs available in the literature include Tanyimboh and Templeman (2004, 2010), Udo and Ozawa (2001), Germanopoulos (1985), Gupta and Bhave (1996), Fujiwara and Ganesharajah (1993), Cullinane et al. (1992) and Wagner et al. (1988). In general, these formulae have been defined on the basis that nodal demand is satisfied in full when the nodal head is equal to or greater than the desired head and zero when the nodal head is equal to or lower than the minimum head. A major advantage of this type of PDA is that the non-linear constitutive equations are solved only once. The work presented herein is based on this approach.

Several PDA works have been carried out based on the HFR approach. Tanyimboh and Templeman (2010) developed a robust algorithm based on the Newton Raphson method. The model was termed as PRAAWDS (Program for the Realistic Analysis of 
the Availability of Water Distribution Systems). Giustolisi et al. (2008b) embedded the Wagner et al. (1988) equation into the Global Gradient Method (GGM) and presented results for two networks which consist of pipes only. The performance of the PDA simulator for analysing networks with pumps and valves was not included. In Giustolisi et al. (2008a), the hydraulic performance of a single source WDS was assessed over 24 hours using PDA with values of the required nodal head (for full demand satisfaction) that varied according to the diurnal demand pattern. However, at least for water utilities within the UK, the prescribed level of service is fixed and does not vary throughout the day or night (OFWAT 2004). Wu et al. (2009) also modified the GGM to incorporate pressure dependent demand. However, unlike EPANET 2, the PDA model used was commercial software the details of which are not in the public domain. Due to space constraints, the literature review presented herein is rather brief. More comprehensive and complete reviews on PDA can be found in Tanyimboh and Templeman (2010) and Wu et al. (2009).

One common weakness in majority of these HFRs is the absence of continuity in the function and/ or their derivatives at the transitions between zero and partial nodal flow and/or between partial and full demand satisfaction. These discontinuities can lead to convergence difficulties in the computational solution of systems of constitutive equations (Tanyimboh and Templeman 2010). By contrast, the Tanyimboh and Templeman (2004, 2010) HFR and its derivative have no discontinuities and is believed to be a reasonable approximation to the node pressure-flow relationship. Also, the derivative for this function can be easily calculated. These characteristics make it ideal to be incorporated effectively into the system of equations used in the EPANET 2 hydraulic engine which is a Newton method based algorithm.

This paper demonstrates the effectiveness of integrating the continuous Tanyimboh and Templeman $(2004,2010)$ function into the Global Gradient Method (Todini and Pilati 1988) to form a model capable of handling real networks involving both normal and pressure deficient conditions. This formulation is referred to as the head dependent Gradient Method (HDGM) and has been successfully implemented within the EPANET 2 (Rossman 2002) framework, extending the renowned DDA hydraulic simulator (Liberatore and Sechi 2009, Cisty 2010, Haghighi et al. 2011) to be able to handle PDA. This seamless enhanced version is termed EPANET-PDX (pressuredependent extension) and is presented herein. EPANET-PDX is capable of simulating real world networks and is able to provide a fully equipped extended period simulation. Results presented herein indicate that EPANET-PDX is robust, accurate, computationally highly efficient and compares very favourably to EPANET 2.

\section{Pressure Dependent Demand Function}

The Tanyimboh and Templeman $(2004,2010)$ head-flow function can be described as

$$
Q n_{i}\left(H n_{i}\right)=Q n_{i}^{r e q} \frac{\exp \left(\alpha_{i}+\beta_{i} H n_{i}\right)}{1+\exp \left(\alpha_{i}+\beta_{i} H n_{i}\right)}
$$

where $Q n_{i}$ and $H n_{i}$ are the nodal flow and head, respectively, at demand node $i . Q n_{i}^{\text {req }}$ is the required supply at node $i$. Both $\alpha_{i}$ and $\beta_{i}$ are parameters to be calibrated with 
relevant field data. However, in the absence of these data, default values of $\alpha_{i}$ and $\beta_{i}$ can be obtained as follows

$$
\begin{aligned}
Q n_{i}\left(H n_{i}^{r e q}\right) & =0.999 Q n_{i}^{r e q} \\
Q n_{i}\left(H n_{i}^{\text {min }}\right) & =0.001 Q n_{i}^{r e q}
\end{aligned}
$$

where $H n_{i}^{\text {min }}$ is the nodal head below which outflow is zero. $H n_{i}^{\text {req }}$ represents the required nodal head for full demand satisfaction. Eq. 2 and Eq. 3 above describe the conditions for virtually full and zero demand satisfaction respectively. Simultaneously solving both equations will give

$$
\begin{gathered}
\alpha_{i}=\frac{-4.595 H n_{i}^{\text {req }}-6.907 H n_{i}^{\min }}{H n_{i}^{r e q}-H n_{i}^{\min }} \\
\beta_{i}=\frac{11.502}{H n_{i}^{\text {req }}-H n_{i}^{\text {min }}}
\end{gathered}
$$

The basic form of the function is illustrated in Fig. 1 in which the demand satisfaction ratio (DSR) is $Q n_{i}\left(H n_{i}\right) / Q n_{i}^{r e q}$. It is worth observing the smooth transition between zero and partial nodal flow and between partial and full demand satisfaction. Further details of this function can be found in Tanyimboh and Templeman (2010).

(Fig.1 here)

\section{Pressure Dependent Demand Model}

This section describes the extension of the Global Gradient Method (GGM) to include demands that are pressure dependent. In the original GGM, the two conservation equations, namely mass balance at nodes and energy conservation along hydraulic links are solved simultaneously as

$$
\left[\begin{array}{ccc}
\mathbf{A}_{11} & \vdots & \mathbf{A}_{12} \\
\cdots & \cdots & \cdots \\
\mathbf{A}_{21} & \vdots & \mathbf{0}
\end{array}\right]\left[\begin{array}{c}
\mathbf{Q p} \\
\cdots \\
\mathbf{H n}
\end{array}\right]=\left[\begin{array}{c}
-\mathbf{A}_{10} \mathbf{H}_{0} \\
\cdots \\
-\mathbf{Q n}^{\text {req }}
\end{array}\right]
$$

where $\mathbf{A}_{11}$ represents the diagonal matrix whose elements are the $\left(K_{j}\left(Q p_{j}\right)^{n}+m Q p_{j}^{2}\right) / Q p_{j}$ for pipes and $-\omega^{2}\left(h_{0}-K_{j}\left(Q p_{j} / \omega\right)^{n}\right) / Q p_{j}$ for pumps. $K_{j}$ and $n$ are the resistance coefficient and flow exponent in the head loss formula respectively. $h_{0}$ is the shutoff head for the pump. $m$ and $\omega$ are the minor loss and pump curve coefficients respectively. $Q p_{j}$ is the flow rate in pipe $j$. The overall incidence matrix relating the pipes to nodes with unknown and known heads is represented by $\mathbf{A}_{12}$ and $\mathbf{A}_{10}$ respectively. Pipe flow leaving node is defined as -1, pipe flow into node as +1 and 0 if pipe is not connected to node. $\mathbf{A}_{\mathbf{2 1}}$ is the transpose of $\mathbf{A}_{12}$. Qp denotes the column vector of unknown pipe flow rates. $\mathbf{H n}$ and $\mathbf{H}_{\mathbf{0}}$ are 
column vectors for unknown and known nodal heads respectively. $\mathbf{Q n}^{\text {req }}$ is the column vector for required nodal supplies.

The continuity equation from Eq. 6a, i.e. $\mathbf{A}_{21} \mathbf{Q p}+\mathbf{Q} \mathbf{n}^{\text {req }}=0$ shows that the sum of flows flowing in and out of the demand node (i.e. $\mathbf{A}_{21} \mathbf{Q p}$ ) is always equal to the required nodal supply. In other words, the nodal demand is assumed to be fully satisfied at all times. In pressure dependent analysis, the nodal outflow is pressure dependent and will not be fully satisfied if the available pressure is insufficient. Hence, the sum of pipe flows in and out of the demand node will not always be equal to the required supply. To incorporate pressure dependent demand, $\mathbf{Q} \mathbf{n}^{\text {req }}$ is replaced with $\mathbf{Q n}(\mathbf{H n})$ where $\mathbf{Q n}(\mathbf{H n})$ represents the pressure dependent nodal flow which is estimated using the Tanyimboh and Templeman (2004) function herein. A diagonal matrix $\mathbf{A}_{22}$ is introduced into Eq. 6a to form

$$
\left[\begin{array}{ccc}
\mathbf{A}_{11} & \vdots & \mathbf{A}_{12} \\
\cdots & \cdots & \cdots \\
\mathbf{A}_{21} & \vdots & \mathbf{A}_{22}
\end{array}\right]\left[\begin{array}{l}
\mathbf{Q p} \\
\cdots \\
\mathbf{H n}
\end{array}\right]=\left[\begin{array}{c}
-\mathbf{A}_{10} \mathbf{H}_{0} \\
\cdots \\
0
\end{array}\right]
$$

where the elements of the diagonal matrix $\mathbf{A}_{22}$ are $\mathbf{Q n}(\mathbf{H n}) / \mathbf{H n}$.

Eq. $6 b$ is then differentiated with respect to the pipe discharges and nodal heads to give

$$
\left[\begin{array}{ccc}
D_{11} & \vdots & A_{12} \\
\cdots & \cdots & \cdots \\
A_{21} & \vdots & D_{22}
\end{array}\right]\left[\begin{array}{c}
d Q p \\
\cdots \\
d H n
\end{array}\right]=\left[\begin{array}{c}
d E \\
\cdots \\
d q
\end{array}\right]
$$

where the elements of diagonal matrix $\mathbf{D}_{\mathbf{1 1}}$ can be written as $n K_{j}\left|Q p_{j}\right|^{n-1}+2 m\left|Q p_{j}\right|$ for pipes and $n \omega^{2} K_{j}\left(Q p_{j} / \omega\right)^{n-1}$ for pumps. $\mathbf{D}_{22}$ represents a diagonal matrix whose elements are the derivatives of $\mathbf{Q n}(\mathbf{H n})$ which is expressed as $Q n_{i}^{\text {req }} \cdot \beta \cdot \exp \left(\alpha_{i}+\beta_{i} H n_{i}\right) \cdot\left(1+\exp \left(\alpha_{i}+\beta_{i} H n_{i}\right)\right)^{-2}$. dQp and $\mathbf{d H n}$ represent the corrective steps for $\mathbf{Q p}$ and $\mathbf{H n}$, respectively, in successive iterations and can be defined as

$$
\begin{aligned}
& \mathbf{d Q p}=\mathbf{Q} \mathbf{p}^{k+1}-\mathbf{Q} \mathbf{p}^{k} \\
& \mathbf{d H n}=\mathbf{H n}^{k+1}-\mathbf{H n}^{k}
\end{aligned}
$$

in which $k$ represents the iteration number. The computational solution scheme for Eq. $6 \mathrm{~b}$ is based on successive linearization using a first order Taylor series expansion from which it can be shown that $\mathbf{d E}$ and $\mathbf{d q}$ represent the energy conservation and mass balance equations respectively. Therefore, with reference to Eq. 6b,

$$
\mathbf{d E}=\mathbf{A}_{11} Q \mathbf{p}+\mathbf{A}_{12} \mathbf{H n}+\mathbf{A}_{10} \mathbf{H}_{0}
$$




$$
\mathbf{d q}=\mathbf{A}_{21} \mathbf{Q p}+\mathbf{A}_{22} \mathbf{H n}
$$

By substituting Eq. 10 and Eq. 11 into Eq. 7, the iterative procedure for solving Eq. $6 \mathrm{~b}$ is obtained as follows.

$$
\begin{gathered}
\mathbf{H} \mathbf{n}^{k+1}=\mathbf{A}^{-1} \mathbf{F} \\
\mathbf{F}=\mathbf{A}_{\mathbf{2 1}} \mathbf{Q} \mathbf{p}^{k}+\mathbf{Q n}\left(\mathbf{H} \mathbf{n}^{k}\right)-\mathbf{D}_{\mathbf{2 2}} \mathbf{H} \mathbf{n}^{k}-\mathbf{A}_{\mathbf{2 1}} \mathbf{D}_{\mathbf{1 1}}^{-1} \mathbf{A}_{\mathbf{1 1}} \mathbf{Q} \mathbf{p}^{k}-\mathbf{A}_{\mathbf{2 1}} \mathbf{D}_{\mathbf{1 1}}^{-1} \mathbf{A}_{\mathbf{1 0}} \mathbf{H}_{\mathbf{0}} \\
\mathbf{A}=\mathbf{A}_{\mathbf{2 1}} \mathbf{D}_{\mathbf{1 1}}^{-1} \mathbf{A}_{\mathbf{1 2}}-\mathbf{D}_{\mathbf{2 2}} \\
\mathbf{Q} \mathbf{p}^{k+1}=\mathbf{Q} \mathbf{p}^{k}-\mathbf{D}_{\mathbf{1 1}}^{-1}\left(\mathbf{A}_{\mathbf{1 1}} \mathbf{Q} \mathbf{p}^{k}+\mathbf{A}_{\mathbf{1 2}} \mathbf{H} \mathbf{n}^{k+1}+\mathbf{A}_{\mathbf{1 0}} \mathbf{H}_{\mathbf{0}}\right)
\end{gathered}
$$

Hence the algorithm first updates the nodal heads $\mathbf{H n}$ by calculating $\mathbf{H n}^{k+1}$ (Eqs. 1214) before updating the pipe flow rates $\mathbf{Q p}$ by calculating $\mathbf{Q p}{ }^{k+1}$ (Eq. 15). Detailed derivations of Eqs. 12-15 and further details regarding matrices $\mathbf{A}_{11}, \mathbf{A}_{12}, \mathbf{A}_{21}$ and $\mathbf{A}_{10}$ can be found in Rossman (2002) and Salgado et al. (1993).

\section{Backtracking and Line Search Procedure}

The integration of the HFR into the system of hydraulic equations is really a complicated task. One major challenge encountered in doing so is the deterioration of the GGM algorithm's excellent convergence property. To include a HFR into the mass balance equations would further increase the overall non-linearity of the system of equations and render it more complex and difficult to solve. Directly applying the corrective steps obtained from the iterative solution methods for non-linear systems of equations would not be sufficient. For example, Newton's method often fails to converge if the starting point (i.e. the initial estimate) is not close to a solution. A globally convergent strategy that yields a solution from almost any starting point is essentially required.

Giustolisi et al. (2008a) adopted a heuristic approach in their pressure-driven network simulation model whereby an over-relaxation parameter that adjusts the Newton step consisting of both pipe-flow and nodal-head corrections is increased or decreased depending on the errors in the mass and energy balance equations. In the Head-Driven Simulation Model developed by Tabesh et al. (2002), convergence is ensured by using a step length adjustment parameter. However, its value is obtained by means of trial and error.

Tanyimboh and Templeman (2010) utilized the backtracking and line search routine in their PDA program PRAAWDS to guide the Newton search in the right direction and ensure global convergence for the system of non-linear equations. It determines the appropriate Newton step size in a deterministic manner, ensuring both the energy and mass conservation functions are sufficiently improved in successive iterations. No trial runs or parameter calibrations were required. Experience with PRAAWDS has shown that the backtracking and line search routine is efficient and reliable. For this 
reason, EPANET-PDX has utilised this technique in enhancing its convergence properties.

The backtracking and line search routine used herein has been adapted from Press et al. (1992). The following section describes our implementation of the backtracking and line search procedure in the integration of the Tanyimboh and Templeman (2004) nodal head-flow function within the GGM. Equations 10 and 11 for conservation of energy and conservation of mass, respectively, can be re-written as

$$
\begin{gathered}
\operatorname{dE}(Q p, H n)=A_{11} Q p+A_{12} H n+A_{10} H_{0} \\
\operatorname{dq}(Q p, H n)=A_{21} Q p+A_{22} H n
\end{gathered}
$$

Together, Eq. 16 and Eq. 17 form a single system of simultaneous nonlinear equations hereinafter referred to as $\mathbf{G}(\mathbf{Q p}, \mathbf{H n})$ the solution of which is required. Accordingly, $\mathbf{G} \equiv[\mathbf{d E}: \mathbf{d q}]^{T}$ and, from Eq. $6 \mathrm{~b}$, it can be seen that $\mathbf{G}(\mathbf{Q p}, \mathbf{H n})=\mathbf{0}$ at the solution. The aim of the backtracking and line search procedure is to ensure that the function $g(\mathbf{Q p}, \mathbf{H n})=\mathbf{G}^{T} \mathbf{G}$ decreases sufficiently in each iteration of the computational solution algorithm so that, at the solution, $g=0$.

In order to incorporate the backtracking and line search procedure the nodal heads $\mathbf{H n}$ are updated iteratively as

$$
\mathbf{H n}^{k+1}=\mathbf{H n}^{k}+\lambda \cdot \boldsymbol{\delta H n}
$$

where the scalar parameter $\lambda$ is an over-relaxation coefficient that satisfies $0<\lambda \leq 1$ and $k$ represents the iteration number. $\mathbf{\delta H n}$ is the full Newton step for the nodal heads Hn. Upon substituting the newly obtained nodal heads $\mathbf{H n}^{k+1}$ into Eq. 15, the new pipe flows $\mathbf{Q} \mathbf{p}^{k+1}$ are obtained.

To ensure that the function $g$, a scalar, has decreased sufficiently, a prescribed minimum reduction in $g$ is enforced to ensure that at least a fraction $\varepsilon$ of the initial rate of reduction in $g$ in the Newton direction $\boldsymbol{\delta}=[\boldsymbol{\delta Q \mathbf { p }}: \mathbf{\delta H n}]^{T}$ is achieved. The initial rate of reduction in $g$ at the current iterate $\left[\mathbf{Q p ^ { k }}: \mathbf{H n}^{k}\right]^{T}$ is $\nabla \mathbf{g}^{T}[\boldsymbol{\delta} \mathbf{Q p}: \boldsymbol{\delta} \mathbf{H n}]^{T}$ in which $\nabla \mathbf{g}$ is the gradient of $g$. Therefore, the acceptance criterion for the next iterate $\left[\mathbf{Q p}{ }^{k+1}: \mathbf{H n}^{k+1}\right]^{T}$ is

$$
g\left(\mathbf{Q} \mathbf{p}^{k+1}, \mathbf{H} \mathbf{n}^{k+1}\right) \leq g\left(\mathbf{Q} \mathbf{p}^{k}, \mathbf{H n}^{k}\right)+\varepsilon\left[\nabla \mathbf{g}\left(\mathbf{Q} \mathbf{p}^{k}, \mathbf{H n}^{k}\right)^{T}\right]^{T}\left[\left(\mathbf{Q} \mathbf{p}^{k+1}-\mathbf{Q} \mathbf{p}^{k}\right) \vdots\left(\mathbf{H n}^{k+1}-\mathbf{H n}^{k}\right)\right]^{T}
$$

where the scalar parameter $\varepsilon$ satisfies $0<\varepsilon<1$; we used $\varepsilon=10^{-4}$ (Press et al. 1992).

In EPANET-PDX, to exploit the quadratic convergence of the GGM algorithm near the solution, the full Newton step $\boldsymbol{\delta}=[\boldsymbol{\delta Q \mathbf { p }}: \boldsymbol{\delta} \mathbf{H n}]^{T}$, i.e. $\lambda=1$ in Eq. 18 , is always tried first. However, if the full Newton step given by $\lambda=1$ in Eq. 18 is unsatisfactory, i.e. $g\left(\mathbf{Q} \mathbf{p}^{k+1}, \mathbf{H} \mathbf{n}^{k+1}\right)$ does not meet the acceptance criterion in Eq. 19, that is to say 
$g\left(\mathbf{Q} \mathbf{p}^{k}, \mathbf{H n}^{k}\right)-g\left(\mathbf{Q} \mathbf{p}^{k+1}, \mathbf{H} \mathbf{n}^{k+1}\right)$ is not large enough and thus $g$ has not decreased sufficiently, backtracking along the Newton direction $\boldsymbol{\delta}$ is carried out. The first backward step or backtrack along the Newton direction $\boldsymbol{\delta}$ is effected by modelling $g\left(\mathbf{Q} \mathbf{p}^{k+1}, \mathbf{H n}^{k+1}\right) \equiv g(\lambda)$ as a quadratic function of $\lambda$ using the substitution $\mathbf{H n}^{k+1}=$ $\mathbf{H n}^{k}+\lambda \cdot \boldsymbol{\delta} \mathbf{H n}$. The value of $\lambda$ that minimizes the function $\mathrm{g}$ is then obtained which, therefore, yields $\mathbf{H n}^{k+1}$ and $\mathbf{Q} \mathbf{p}^{k+1}$. If more backtracking is necessary, then, for the second and any subsequent backtracks, $g(\lambda)$ is modelled as a cubic function of $\lambda$. The backtracking procedure continues until either Eq. 19 is satisfied or $\lambda$ reaches the minimum set value $\lambda_{\min }$. We set a minimum value of $\lambda_{\min }=0.2$ to stop the algorithm from taking steps that are too small as this would result in a large number of iterations and consequently a longer computational time.

The backtracking and line search procedure can be summarized with the following flow chart presented in Fig. 2. Overall, this strategy has proven to be robust and efficient in leading the HDGM algorithm to convergence. Without this global convergence strategy, the HDGM algorithm is prone to oscillate without converging even while analyzing trivial networks.

(Fig. 2 here)

\section{Convergence Criteria}

The convergence criteria were chosen such that the absolute values of the maximum changes in both the nodal heads and pipe flows would be less than $0.001 \mathrm{ft}$ $\left(3.048 \times 10^{-4} \mathrm{~m}\right)$ and $0.001 \mathrm{cfs}\left(2.832 \times 10^{-5} \mathrm{~m}^{3} \mathrm{~s}^{-1}\right)$ respectively, i.e.

$$
\|\boldsymbol{\delta}\|_{\infty} \leq 10^{-3}
$$

in which $\boldsymbol{\delta}=[\boldsymbol{\delta} \mathbf{Q p}: \delta \mathbf{H n}]^{T}$ is in cfs and ft, respectively. These criteria may be more stringent compared to the default one used in EPANET 2. The criterion used in EPANET 2 is the ratio of the sum of the absolute values of pipe flow changes to the total flow in all pipes which should be less than 0.001. However, computational experience has shown that the above mentioned criteria in Eq. 20 (i.e. the absolute value of each component of $\boldsymbol{\delta Q p} \leq 0.001 \mathrm{cfs}$ and the absolute value of each component of $\boldsymbol{\delta} \mathbf{H n} \leq 0.001 \mathrm{ft}$ ) prevent spurious convergence and enable the algorithm to perform more consistently. Similarly to EPANET 2, a maximum of 200 iterations is also used as a further threshold control for convergence.

\section{Case Studies}

To illustrate the application of EPANET-PDX, a total of 420 steady state simulations and 30 EPSs were performed over six networks of different sizes. Each EPS had a duration of 24 hours and a 1 hour hydraulic time step. Simulations executed involved 
1) Varying the source heads thus subjecting the networks to the entire range of DSRs; and

2) Randomly closing pipes to create stress within the networks.

Table 1 shows the characteristics of the networks along with the numbers and types of simulation carried out. Under the pipe closure (PC) simulation column in Table 1, values in parentheses represent the number of pipes closed. For example, the number of pipes closed for network 2 ranged from 1 pipe to 8 pipes. Also, EPANET 2 was run concurrently for each simulation to serve as comparison for both PDA and DDA. Thus overall, 840 steady state and 60 EPS simulations were involved in this assessment. Additional EPANET 2 simulations were carried out to verify the accuracy of the PDA results as detailed later in the "results verification" in Section 8.

(Table 1 here)

Overall, the performances of EPANET-PDX and EPANET 2 were very similar as shown herein. Consequently, not all aspects of results will be presented for every network. However, detailed results of the simulators' performance on the whole in terms of robustness, average CPU time and number of iterations are presented and discussed at the end of the paper.

It is essential to clarify two key terms which will be extensively used in the following section of the paper. The term demand satisfaction ratio (DSR) represents the ratio of the available nodal flow to the nodal demand and takes values between 0 and 1 . A network DSR value of 0.5 means only $50 \%$ of the total network demand is satisfied. It is also worth mentioning that DSRs for nodes and networks are only presented for EPANET-PDX and not EPANET 2. The reason is EPANET 2 is a DDA based hydraulic simulator and hence the nodal demands are implicitly assumed to be satisfied in full regardless of whether the pressure is sufficient or not. The second term, nodal residual pressure head refers to the pressure head of the node excluding the elevation.

\subsection{Network 1}

The first example shown in Fig. 3 is based on a simple benchmark network taken from the literature. This single source network consists of 8 pipes of length $1000 \mathrm{~m}$ and 6 demand nodes with desired heads of $60 \mathrm{~m}$. More pipe and node data are available in Alperovits and Shamir (1977). EPANET-PDX was carried out with a variation of source head from $37 \mathrm{~m}$ to $84 \mathrm{~m}$. The nodal heads and pipe flows obtained were essentially identical to Tanyimboh and Templeman (2010).

(Fig. 3 here)

Pipe closure (PC) simulations were executed on this network by closing different individual pipes at a time. The source head was set to $79 \mathrm{~m}$. Based on the PDA results, 
the exact shortfall in network performance due to the pipe closure can be accurately quantified as shown in Fig. 4. It can be observed that closing pipes nearer to the source has greater effect on the entire network performance as expected.

(Fig. 4 here)

\subsection{Network 2}

The WDS layout of the second example is shown in Fig. 5. Further details of pipes and nodes can be found in Reddy and Elango (1989). The effective source head was varied from $5 \mathrm{~m}$ to $40 \mathrm{~m}$. The detailed EPANET-PDX results can be found in Siew and Tanyimboh (2011a).

(Fig. 5 here)

A comparison of computational performance achieved using EPANET-PDX and EPANET 2 for source head variation (SHV) simulations is summarized in Fig. 6. Both simulators achieved an identical average of 4.3056 iterations per simulation. In the aspect of CPU time, EPANET-PDX achieved a slightly lower average value of 0.0640 s per simulation as compared to $0.0673 \mathrm{~s}$ by EPANET 2 .

(Fig. 6 here)

\subsection{Network 3}

The third network is based on Jeppson and Davis (1976). The network has two sources, two pumps and one pressure reducing valve as shown in Fig. 7. Pipe and node data are given in Fig. 7 and Table 2 respectively. The hydraulic characteristics of pumps 10 and 11 were represented by $h_{p}=26.67-1042 Q_{p}{ }^{2}$ and $h_{p}=33.33-1029 Q_{p}{ }^{2}$ respectively where $h_{p}$ is the head supplied by the pump in $\mathrm{m}$ and $Q_{p}$ is the pump discharge in 1/s. The pressure-reducing valve (PRV) 12 was set to $140 \mathrm{~m}$. The desired residual heads of the demand nodes were each set to $20 \mathrm{~m}$. Nodes $1,3,7$ and 8 are dummy nodes. A total of 72 SHV simulations with different network conditions were performed by simultaneously decreasing the head at both sources 10 and 11 from $158 \mathrm{~m}$ to $14 \mathrm{~m}$ and from 238 to $94 \mathrm{~m}$ respectively.

(Fig. 7 here)

(Table 2 here) 
A closer examination of node 4 was carried out. It is important to restate that the DSR results presented in these figures correspond to the heads generated by EPANETPDX, and not EPANET 2. Based on the results in Fig. 8, DDA generates significantly lower nodal heads during pressure deficient scenarios, i.e. when nodal residual head is below 20m, giving a very false depiction of the nodal performance. For example, when the head of source 10 was within the range of 90 to $100 \mathrm{~m}$, the DSR of node 4 is approximately in the range of 0.7 to 0.8 , meaning that there is actually substantial flow emitting from the node. However, based on the negative pressure computed by DDA, one might have the impression that there is no flow at all from node 4 within this source head range. Also, it is shown that the gap between DDA and PDA results gradually closes and finally merges as the nodal DSR approaches 1 . This clearly demonstrates that the more pressure deficient a network is, the more DDA results underestimate its performance. This also shows that during normal operating conditions, results generated by both analyses are identical.

(Fig. 8 here)

For SHV simulations, the numbers of iterations were virtually identical. The CPU time required (as shown in Fig. 9) was slightly higher for EPANET-PDX. As for PC simulations, both hydraulic simulators achieved identical iteration counts for every simulation.

(Fig. 9 here)

\subsection{Network 4}

Fig. 10 shows the layout of a real life network supplying water to a mixed rural and suburban area with an approximate population of 15,000. The system consists of 164 nodes, 200 pipes, 5 reservoirs, 4 pumps and 2 flow control valves. Further details of the network can be found in Tanyimboh (2008) and Shan (2004). For all demand nodes, the desired residual head was set to $15 \mathrm{~m}$.

(Fig. 10 here)

In this case study, a pressure deficient condition was created by introducing a pressure shortage (with a water level of $100 \mathrm{~m}$ ) for each reservoir such that only $22 \%$ of the total demand was satisfied. Fig. 11 shows the residual head of each demand node generated by both simulators. Based on EPANET 2 (DDA) results, one might have the impression that only one demand node meets the desired residual head requirement, i.e. $15 \mathrm{~m}$. However, based on EPANET-PDX (PDA) results, a total of seven demand nodes have residual heads above the desired value and are satisfied in full. These seven nodes correspond to a DSR of 1 as shown in Fig. 12. This reinforces the fact that DDA underestimates the capacity of a pressure deficient network. The 
performance of each demand node can be accurately assessed based on the nodal demand satisfaction ratio (DSR) shown in Fig. 12. It is worth mentioning that nodes 1 to 19 have no demand and thus are not shown in both Fig. 11 and Fig. 12.

(Fig. 11 here)

(Fig. 12 here)

\subsection{Network 5}

The fifth example (Fig. 13) is a generic network (Shan, 2004) which consists of 204 nodes, 557 pipes and 2 reservoirs. All nodal elevations, required heads and demands were $75 \mathrm{~m}, 90 \mathrm{~m}$ and $10 \mathrm{l} / \mathrm{s}$ respectively. All pipe lengths, diameters and HazenWilliams roughness coefficients were $100 \mathrm{~m}, 0.45 \mathrm{~m}$ and 130 respectively. A total of 45 PDA simulations were executed with heads at both reservoirs 205 and 206 varying uniformly from $66 \mathrm{~m}$ to $110 \mathrm{~m}$. The network performance is summarised in Fig. 14. The performance of EPANET-PDX remains on a par with EPANET 2 even for large networks as shown in Fig. 15 and Fig. 16.

(Fig. 13 here)

(Fig. 14 here)

(Fig. 15 here)

(Fig. 16 here)

\subsection{Network 6}

Network 6 is the benchmark "Anytown" network (Fig. 17) and was chosen to demonstrate the capability of the pressure dependent EPS. The "Anytown" network originally presented an optimization problem involving the upgrading of the system to meet future demands with options including new pipes, cleaning and lining of existing pipes, construction of new pumping stations and tanks. Hence, several modifications to the original (un-optimized network) input data were made with the sole purpose of enabling an effective EPS to be demonstrated. The diameters of the six new pipes (10, $13,14,15,16$ and 25 ) were set to be $0.3048 \mathrm{~m}$ (12 in). Demands for nodes 2, 4, 5, 9, $10,12,15$ were reduced to $3.155 \mathrm{l} / \mathrm{s}$ (50 GPM). The modified demand factors (DFs) are presented in Fig. 18. The DFs represent the variation in water demand throughout the day. For example, a DF value of 0.6 for the $8^{\text {th }}$ and $9^{\text {th }}$ hours means that the water 
consumption during both these hours is 0.6 times the average water use. The rest of the network data remain the same as used in Walski et al. (1987).

(Fig. 17 here)

The two tanks were operated with water levels between elevations $68.58 \mathrm{~m}(225 \mathrm{ft})$ and $76.2 \mathrm{~m}(250 \mathrm{ft})$. A minimum pressure of at least $28.12 \mathrm{~m} \mathrm{(40psi)} \mathrm{must} \mathrm{be} \mathrm{provided}$ at all nodes. Both tanks were emptied and filled completely over their operational ranges during the day. The network was pressure deficient during the peak demand hours when both tanks were fully depleted. The hydraulic time step used was 1 hour. Observing the plots in Fig. 18, the intermediate results for tank heads and network DSR between successive hydraulic time steps indicate that the tanks are either completely full or empty.

(Fig. 18 here)

Tank 1 and Tank 2 were completely depleted at time 13:27 and 12:37 respectively causing the network to experience a significant drop in DSR as the demand continued to increase with time. Comparing the magnitude of the difference in the nodal heads generated by both analyses at time 16:00 and 19:00 (Fig. 19 and Fig. 20), it is once again shown that the more pressure deficient the network is, the more DDA underestimates its performance. It is worth mentioning that node 1 is connected directly to the source via dummy node 20 and 3 pumps operating in parallel. Hence both these nodes are supplied with constant high pressure throughout the day without being affected much by the variation in demand.

(Fig. 19 here)

(Fig. 20 here)

A total of 20 EPSs were carried out while varying the heads of both tanks simultaneously from $56.388 \mathrm{~m}(150 \mathrm{ft})$ to $85.344 \mathrm{~m}(245 \mathrm{ft})$. Also, 10 additional EPSs with various pipes closed were also executed. Each EPS had a duration of 24 hours and a hydraulic time step of 1 hour. Hence approximately 720 (or more) steady state analyses were performed for this network.

It is worth mentioning that aside from the simulations presented herein, some 46 million simulations have been carried out satisfactorily so far within the Penalty-Free Multi-Objective Evolutionary Algorithm (Siew and Tanyimboh 2010b) framework to solve the WDS benchmarks of Alperovits and Shamir (1977), Hanoi, New York tunnels, Anytown and the Wobulenzi system (Siew and Tanyimboh 2010b, 2011b and 2011c). 


\section{Comparison of Performance of EPANET-PDX and EPANET 2}

Tables 3 and 4 summarize the performances for both EPANET-PDX and EPANET 2 for all six networks simulated. It is worth mentioning that for Network 6 , the average CPU time recorded is the duration of a 24 hour EPS. All steady state simulations were carried out with an Intel single core CPU $3.2 \mathrm{GHz}, 2 \mathrm{~GB}$ RAM desktop except for the pipe closure simulations in Network 5. PC simulations of Network 5 along with the EPS of Network 6 were executed using a more efficient Intel Core 2 Duo CPU 2.66 GHz, 3.23 GB RAM. The similarity between the mean and median values shows that there are no anomalous data present that may artificially distort the mean values. This appears to suggest that EPANET-PDX, like EPANET 2 performs consistently. As a whole, the computational efficiency of EPANET-PDX compares very favourably to EPANET 2. The performance of the PDA model remains efficient and does not deteriorate with the increase in network size and the presence of other hydraulic elements such as pumps and valves. In cases where EPANET-PDX required higher $\mathrm{CPU}$ time such as those reported during pipe closure simulations, the differences were rather trivial. From a numerical and computational efficiency standpoint, this comparison shows that there is little to pay to incorporate pressure dependent demands in the hydraulic analysis. Also, contrary to Xu (2001), based on the examples presented, there is no clear trend suggesting that PDA requires more computational effort when analysing networks under pressure deficiency. Essentially the same conclusion was arrived at by Tanyimboh and Templeman (2004 and 2010).

(Table 3 here)

(Table 4 here)

\section{EPANET-PDX Results Verification}

When analyzing a pressure deficient operating condition, unlike DDA, nodal flows computed by the PDA are less and at times vastly different from the corresponding required demands. Consequently, the accuracy and feasibility of the generated PDA results are often questioned. To monitor the accuracy and consistency of a PDA, Ackley et al. (2001) developed a verification technique which is powerful yet straightforward and simple to implement. In this approach, the nodal flows from the PDA solution are entered into an ordinary DDA program as nodal demands. With all other parameters remaining unchanged, the DDA program is executed. Subject to round off error, the resulting set of DDA based nodal heads and pipe flows will be identical to the corresponding nodal heads and pipe flows generated by the PDA program only if the PDA nodal flows are accurate. This test works brilliantly in 
practice and has been implemented in many studies (e.g. Ackley et al. 2001, Tanyimboh et al. 2003, Kalungi and Tanyimboh 2003, Siew and Tanyimboh 2010a).

Herein, this technique is termed the hydraulic feasibility test (HFT) and is utilized as a means of verifying the PDA results generated by EPANET-PDX. To avoid any potential confusion, the DDA program used for the HFT which in this case is EPANET 2 is termed as EPANET 2 HFT. The HFT was carried out on a representative sample of simulations for all the network simulations. A graph of correlation between nodal heads for Network 2 is shown in Fig. 21, where $R^{2}$ is the statistical correlation coefficient. The agreement between the actual PDA heads (generated by EPANET-PDX) and the DDA heads for PDA nodal flows (generated by EPANET 2 HFT) is excellent. Results for the other networks are reported in Table 5 . For easier reading, the correlation values are presented in the form of $1-R^{2}$. Both sets of nodal heads for each network were essentially identical. The accuracy of the EPANET-PDX PDA results is thus confirmed.

(Fig. 21 here)

(Table 5 here)

Another verification means is to evaluate the Euclidean norm of the right hand side of Eq. 7. At the solution, the norm should approach a value of 0 as an indication of the progress and accuracy of the algorithm. This ensures that the convergence of EPANET-PDX simulations is not spurious and the real solution has been found. Fig. 22 shows a consistent decrease of the norm value at successive iterations for 120 unbiased sampled simulations. It is worth observing that the norm reduces very rapidly in the early iterations and by the $4^{\text {th }}$ iteration, a majority of the norm values for these simulations have decreased significantly. These results strongly demonstrate that the backtracking and line search technique effectively optimizes the algorithm's search for the Hn and Qp vectors, leading to a smooth and rapid convergence.

(Fig. 22 here)

Table 6 reports the maximum and mean values of the norm at the last iteration of all simulations (both SHV and PC) executed for each network. It is worth clarifying that the norm values presented here is based on imperial units, i.e. $\mathrm{cfs}$ and $\mathrm{ft}$ for mass and energy balance respectively. This implies that corresponding values in SI units $\left(\mathrm{m}^{3} \mathrm{~s}^{-1}\right.$ and $\mathrm{m}$ ) would be much smaller. Results presented reinforce the evidence that EPANET-PDX is highly robust and reliable.

(Table 6 here) 


\section{Conclusions}

A comprehensive study on PDA and DDA involving a total of 1385 steady state simulations and 70 24-hour EPSs has been carried out. Results presented herein demonstrate that EPANET-PDX is robust, efficient and accurate in analyzing both normal and pressure deficient conditions. In terms of computational efficiency, the performance of EPANET-PDX compares very favourably to EPANET 2. With this said, one should bear in mind that EPANET 2 results are inaccurate, misleading or infeasible while analysing pressure deficient networks as demonstrated clearly in the paper. This new EPANET-PDX model provides a fully equipped pressure dependent extended period simulation and is capable of simulating real world networks with tanks, pumps and valves. Evidence of its robustness includes the ability to produce realistic, hydraulically consistent results for the entire range of network demand satisfaction from zero to $100 \%$ without any convergence complications. Indeed in all of the cases attempted so far, there is no instance where the program failed to converge.

The accuracy of the generated PDA results has been validated and verified using the hydraulic feasibility test and evaluation of the energy and mass balance errors at the solution. Results presented demonstrate the drawbacks of DDA which include the exaggeration of pressure shortage and the inability to quantify the deficiency of the network performance. From a computational standpoint, the backtracking and line search procedure has proven to be effective in providing robustness and very efficient convergence of the hydraulic simulation model. The development of EPANET-PDX has enabled PDA to be used successfully in WDS optimization and the initial results can be found in Siew and Tanyimboh (2010b).

Given the excellent computational properties of EPANET 2, the results herein demonstrate that the performance of EPANET-PDX is essentially on a par with the conventional demand driven approach. Some issues not addressed in this paper include other pressure-dependent nodal flow functions (i.e. HFRs) and integrated pressure-dependent water quality modelling. Also, a comparison between EPANETPDX and other PDA approaches such as Ackley et al. (2001), Giustolisi et al. (2008a,b) and OOTEN (a public-domain object-oriented toolkit for EPANET) is not included. More work on these and other aspects is indicated.

\section{Acknowledgements}

This research was funded in part by the UK Engineering and Physical Sciences Research Council under Grant Number EP/G055564/1. The authors are grateful to the British Government (Overseas Research Students Award Scheme) and the University of Strathclyde for funding for the first author's $\mathrm{PhD}$ programme. 


\section{References}

Ackley JRL, Tanyimboh TT, Tahar B, Templeman AB (2001) Head-driven analysis of water distribution systems. Water Software Systems: Theory and Applications, Vol. 1, Ulanicki, B., Coulbeck, B. and Rance, J. (eds.), Research Studies Press Ltd, England, ISBN 0863802745, Chapter 3:183-192

Alperovits E, Shamir U (1977) Design of optimal water distribution systems. Water Resources Research 13(6):885-900

Ang WH, Jowitt PW (2006) Solution for water distribution systems under pressuredeficient conditions. Journal of Water Resources Planning and Management, ASCE 132(3):175-182

Bhave PR, (1991) Analysis of Flow in Water Distribution Networks. Lancaster, PA: Technomic Publishing

Cisty M (2010) Hybrid genetic algorithm and linear programming method for leastcost design of water distribution systems. Water Resources Management, 24(1): $1-24$

Christodoulou SE (2010) Water network assessment and reliability analysis by use of survival analysis. Water Resources Management, 25(4): 1229-1238

Cullinane MJ, Lansey KE, Mays LW (1992) Optimisation-availability-based design of water distribution networks. Journal of Hydraulics Engineering 118(3):420-441

Fujiwara O, Ganesharajah T (1993) Reliability assessment of water supply systems with storage and distribution networks. Water Resource Research 29(8):29172924.

Germanopoulos G (1985) A technical note on the inclusion of pressure dependent demand and leakage terms in water supply network models. Civil Engineering Systems 2:171-179

Giustolisi O, Kapelan Z, Savic DA (2008a) Extended period simulation analysis considering valve shutdowns. Journal of Water Resource Planning and Management 134(6):527-537

Giustolisi O, Savic DA, Kapelan Z (2008b) Pressure-driven demand and leakage simulation for water distribution networks. Journal of Hydraulic Engineering 134(5):626-635

Gupta R, Bhave PR (1996) Comparison of methods for predicting deficient network performance. Journal of Water Resource Planning and Management 122(3):214217

Haghighi A, Samani HMV, Samani ZMV (2011) GA-ILP method for optimization of water distribution networks. Water Resources Management 25(7): 1791-1808

Jeppson RW, Davis A (1976) Pressure reducing valves in pipe network analyses. Journal of Hydraulics Division 102(HY7):987-1001

Kalungi P, Tanyimboh TT (2003) Redundancy model for water distribution systems. Reliability Engineering and System Safety 82(3):275-286

Martínez-Rodríguez JB, Montalvo I, Izquierdo J, Pérez-García R (2011) Reliability and Tolerance Comparison in Water Supply Networks. Water Resources Management, 25(5):1437-1448

Liberatore S, Sechi GM (2009) Location and calibration of valves in water distribution networks using a scatter-search meta-heuristic approach. Water Resources Management, 23: 1479-1495

OFWAT (2004) Levels of Service for the Water Industry in England and Wales. 2002-2003 Report. Ofwat Centre, 7 Hill Street, Birmingham B5 4UA, UK 
Park S, Choi CL, Kim JH, Bae CH (2010) Evaluating the economic residual life of water pipes using the proportional hazards model. Water Resources Management, 24(12): 3195-3217

Press WH, Teukolsky SA, Vetterling WT, Flannery BP (1992) Numerical Recipes in FORTRAN: The Art of Scientific Computing. Cambridge University Press, New York, USA

Reddy LS, Elango K (1989) Analysis of water distribution networks with head dependent outlets. Civil Engineering Systems 6(3):102-110

Rossman LA (2002) EPANET 2 User's Manual, Water Supply and Water Resources Division, National Risk Management Research Laboratory, Cincinnati, OH45268

Rossman LA (2007) Discussion of 'Solution for water distribution systems under pressure-deficient conditions'. Journal of Water Resources Planning and Management 133(6):566-567

Salgado R, Rojo J, Zepeda S (1993) Extended gradient method for fully non-linear head and flow analysis in pipe networks. Integrated Computer Applications in Water Supply-- Methods and Procedures for Systems Simulation and Control, 1, pp. $49-60$

Shan N (2004) Head dependent modelling of water distribution network. MSc Dissertation, University of Liverpool, UK

Siew C, Tanyimboh TT (2010a) Pressure-dependent EPANET extension: pressuredependent demands. Proceedings of the $12^{\text {th }}$ Annual Water Distribution Systems Analysis Conference, WDSA 2010, September 12-15, Tucson, Arizona

Siew C, Tanyimboh TT (2010b) Penalty-Free Multi-Objective Evolutionary Optimization of Water Distribution Systems. Proceedings of the $12^{\text {th }}$ Annual Water Distribution Systems Analysis Conference, WDSA 2010, September 12-15, Tucson, Arizona

Siew C, Tanyimboh TT (2011a) The computational efficiency of EPANET-PDX. Proceedings of the $13^{\text {th }}$ Annual Water Distribution Systems Analysis Conference, WDSA 2011, May 22-26, Palm Springs, California

Siew C, Tanyimboh TT (2011b) Design of the "Anytown" network using the penaltyfree multi-objective evolutionary optimization approach. Proceedings of the $13^{\text {th }}$ Annual Water Distribution Systems Analysis Conference, WDSA 2011, May 2226, Palm Springs, California

Siew C, Tanyimboh TT (2011c) Penalty-free evolutionary algorithm optimization for the long term rehabilitation and upgrading of water distribution systems. Proceedings of the $13^{\text {th }}$ Annual Water Distribution Systems Analysis Conference, WDSA 2011, May 22-26, Palm Springs, California

Tabesh M, Tanyimboh TT, Burrows R (2002) Head driven simulation of water supply networks. Int. J. Eng., Transactions A: Basics 15(1):11-22

Tabesh M, Yekta A, Burrows R (2009) An integrated model to evaluate losses in water distribution systems. Water Resources Management, 23(3):477-492

Tanyimboh TT (2008) Robust algorithm for head-dependent analysis of water distribution systems. Proceedings of the $10^{\text {th }}$ Annual Water Distribution Systems Analysis Conference, Van Zyl, J.E., Ilemobade, A.A. and Jacobs, H.E. (eds.), August 17-20, Kruger National Park, South Africa

Tanyimboh TT, Tahar B, Templeman AB (2003) Pressure-driven modelling of water distribution systems. Water Science and Technology-Water Supply, 3(1-2):255262

Tanyimboh TT, Templeman AB (2004) A new nodal outflow function for water distribution networks. Proceedings of the $4^{\text {th }}$ International Conf. on Eng. 
Computational Technology, Topping, B.H.V. and Mota Soares, C.A. (eds.), CivilComp Press, Stirling, UK, ISBN 0-948749-98-9, Paper 64, pp. 12, CD-ROM

Tanyimboh TT, Templeman AB (2010) Seamless pressure-deficient water distribution system model. J. Water Management, ICE, 163(8):389-396

Todini E, Pilati S (1988) A gradient algorithm for the analysis of pipe networks. Computer Applications in Water Supply, Volume 1, Coulbeck, B., and Orr, C-H (eds.), Research Studies Press, England

Udo A, Ozawa T (2001) Steady-state flow analysis of pipe networks considering reduction of flow in the case of low water pressures. Water Software Systems: Theory and Applications (Ulanicki B, Coulbeck B and Rance J (eds.)). Research Studies Press, Taunton, UK, Vol. 1, pp. 73-182

Wagner JM, Shamir U, Marks DH (1988) Water distribution reliability: simulation methods. Journal of Water Resources Planning and Management, 114(3):276-294

Walski TM, Brill ED, Gessler J, Goulter IC, Jeppson RM, Lansey K, Lee HL, Liebman JC, Mays L, Morgan DR, Ormsbee L (1987) Battle of the network models: epilogue. Journal of Water Resource Planning and Management, 113(2):191-203

Wu YW, Wang RH, Walski TM, Yang SY, Bowdler D, Baggett CC (2009) Extended global-gradient algorithm for pressure-dependent water distribution analysis. Journal of Water Resource Planning and Management, 135(1):13-22

Xu C (2001) Re: modelling demands. www.bossintl.com/forums/showthr.../thread id/5176.htm, June 2001, accessed 2002 by Tanyimboh and Templeman (2004) 


\section{Figures}

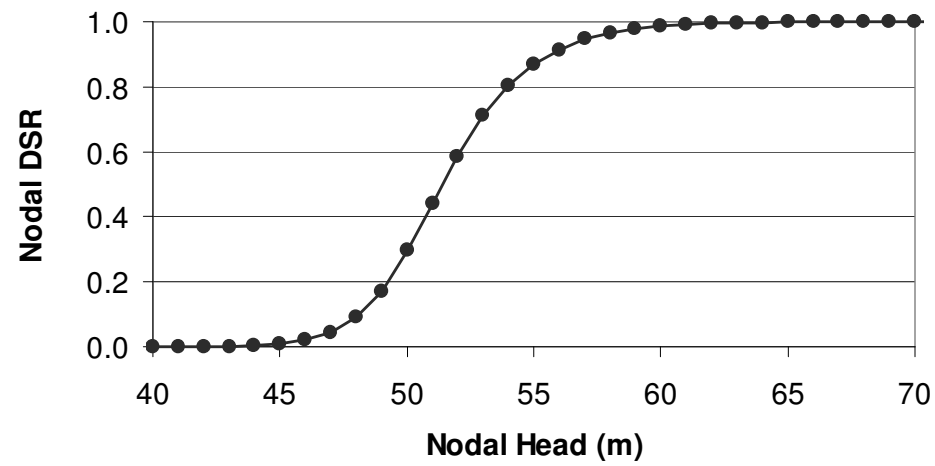

Fig. 1 Pressure-dependent demand function (node elevation $=45 \mathrm{~m}$, desired residual head $=15 \mathrm{~m}$ )

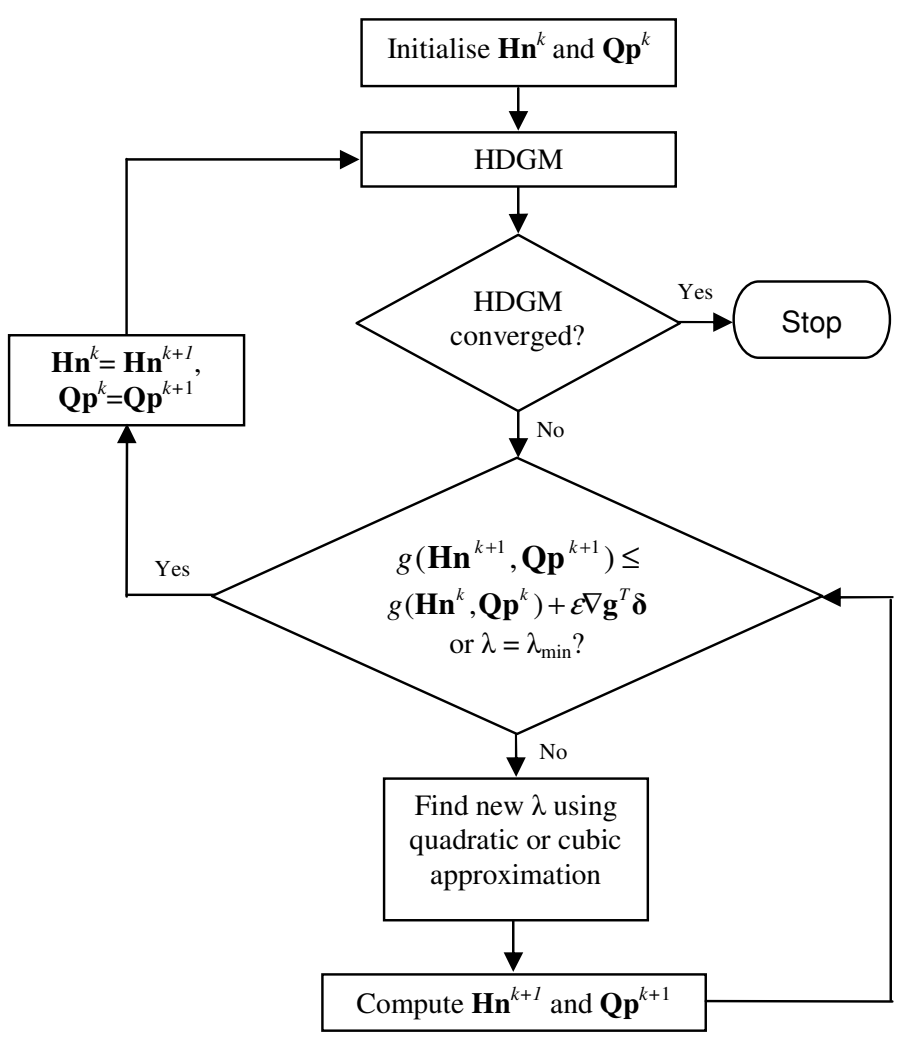

Fig. 2 Line search procedure 


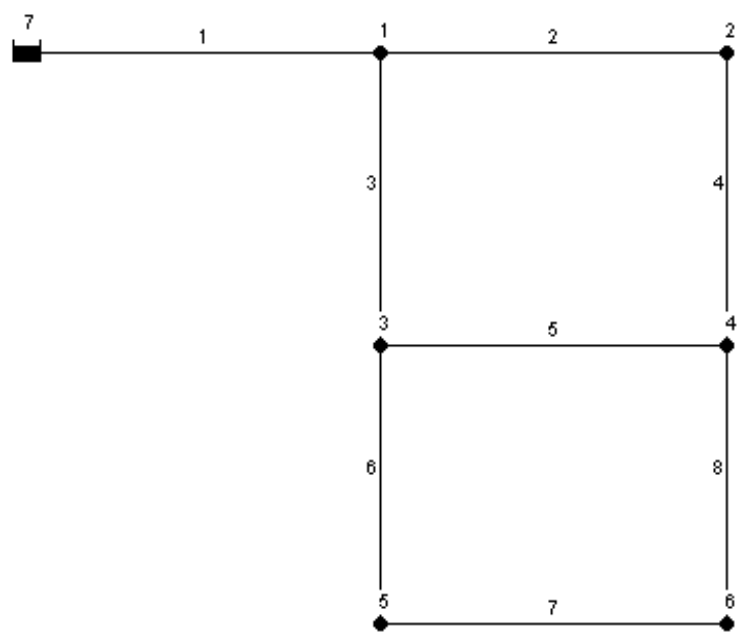

Fig. 3 Layout of Network 1

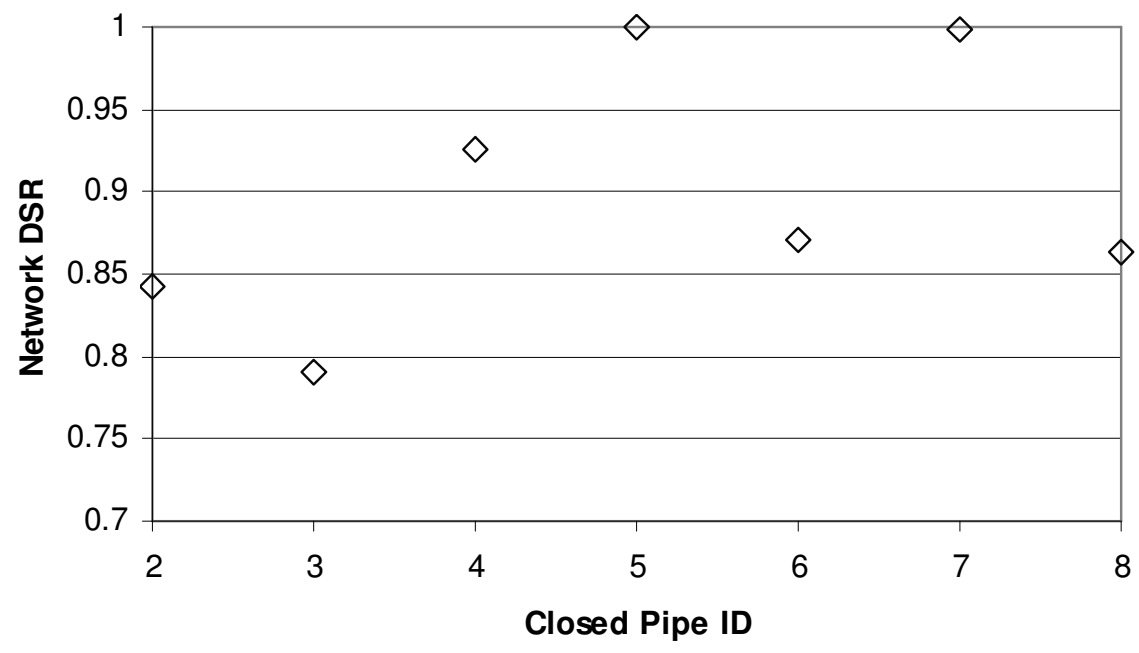

Fig. 4 Network performance for pipe closure simulations 


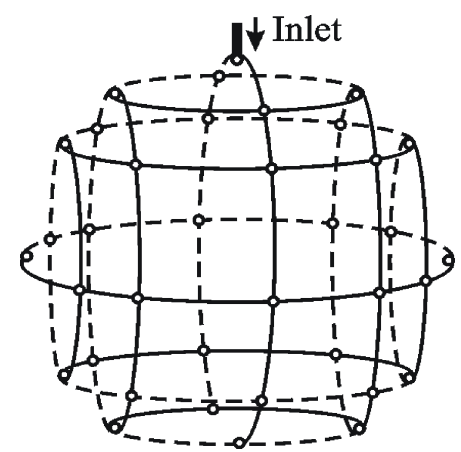

Fig. 5 Layout of Network 2

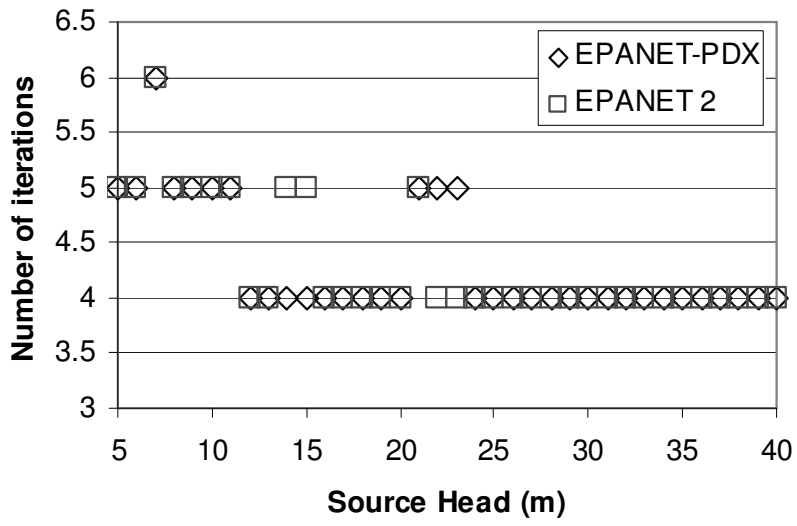

Fig. 6 Number of iterations required by EPANET-PDX and EPANET 2

[Diameter (mm), Length (m), C]

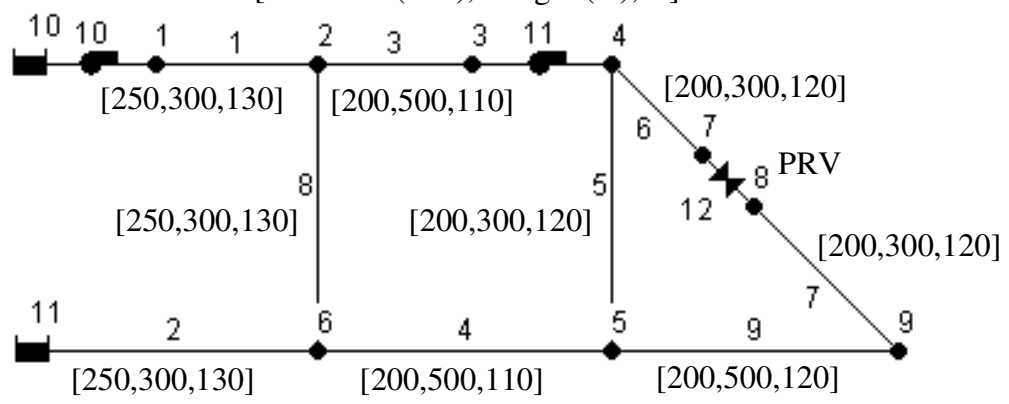

Fig. 7 Layout of Network 3 


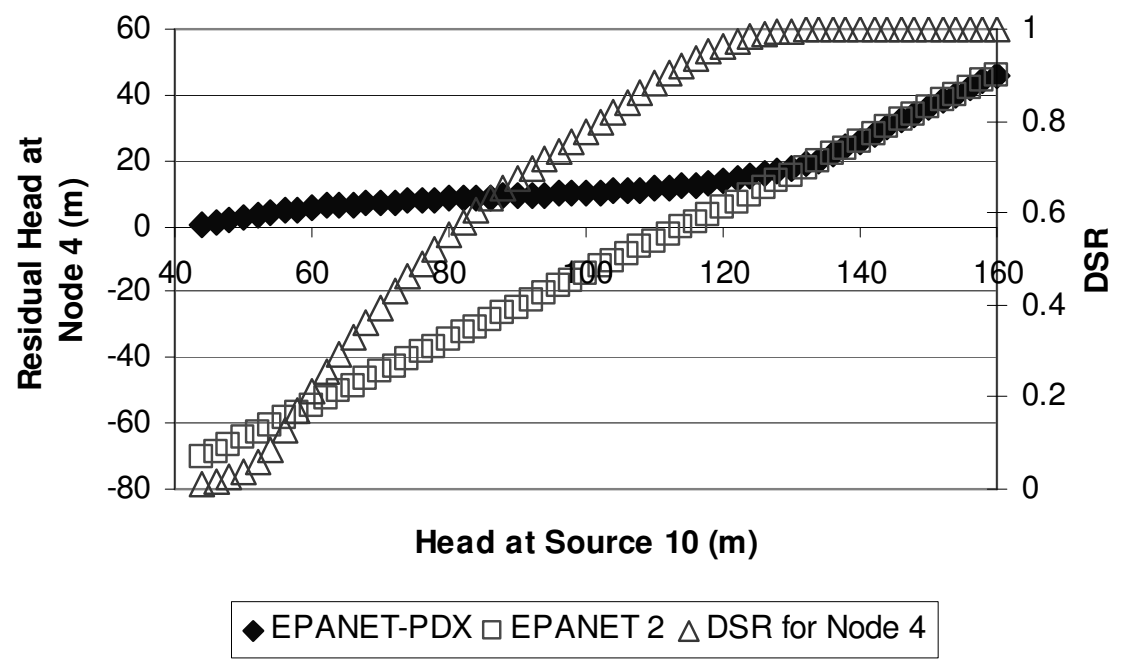

Fig. 8 Residual heads and DSRs of Node 4

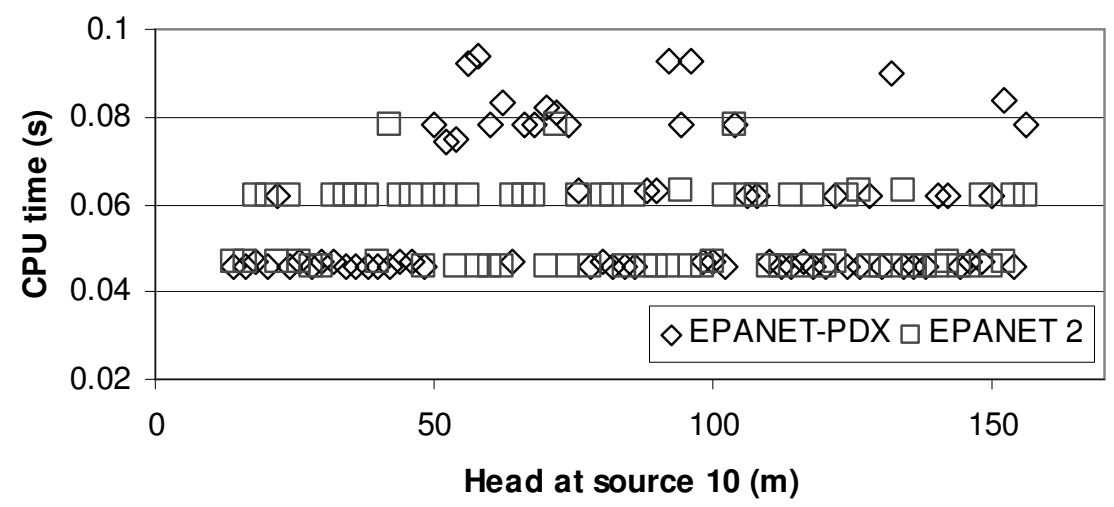

Fig. 9 CPU time required by EPANET-PDX and EPANET 2 


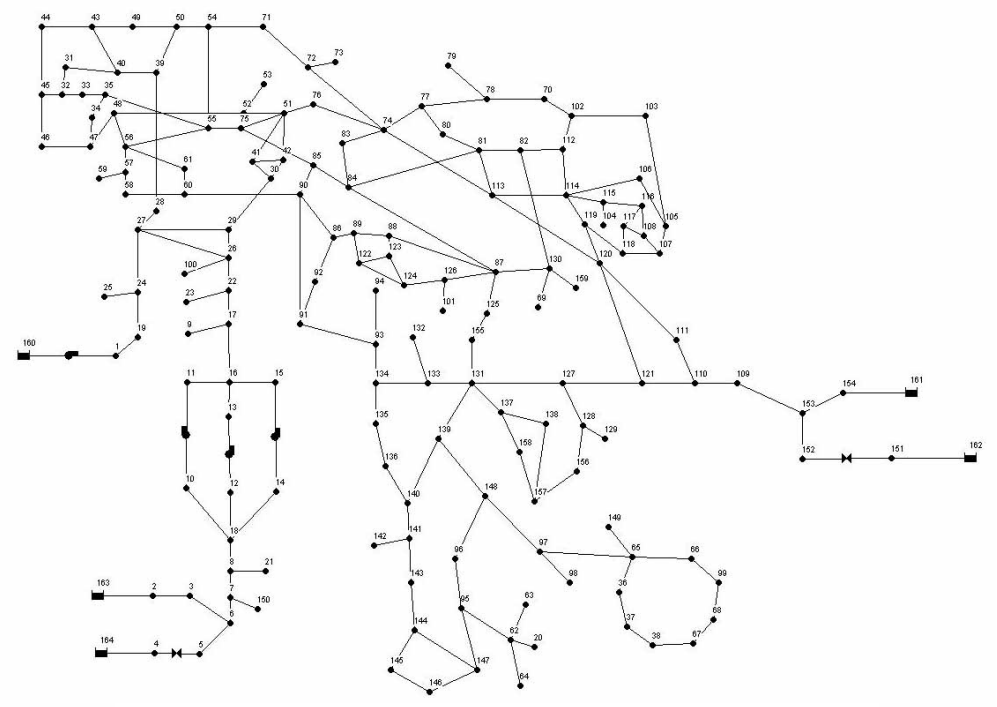

Fig. 10 Layout of Network 4

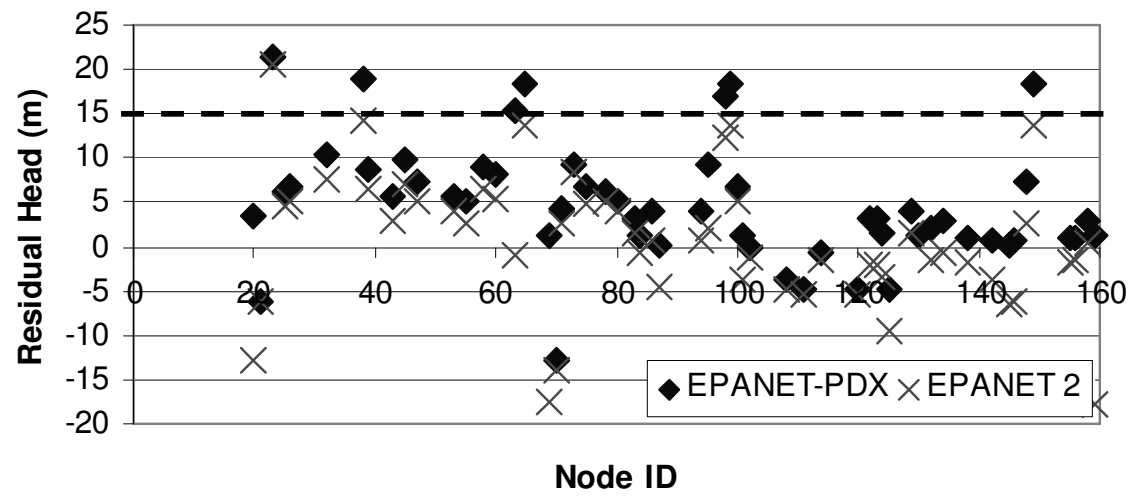

Fig. 11 Nodal residual heads generated by EPANET-PDX and EPANET 2 


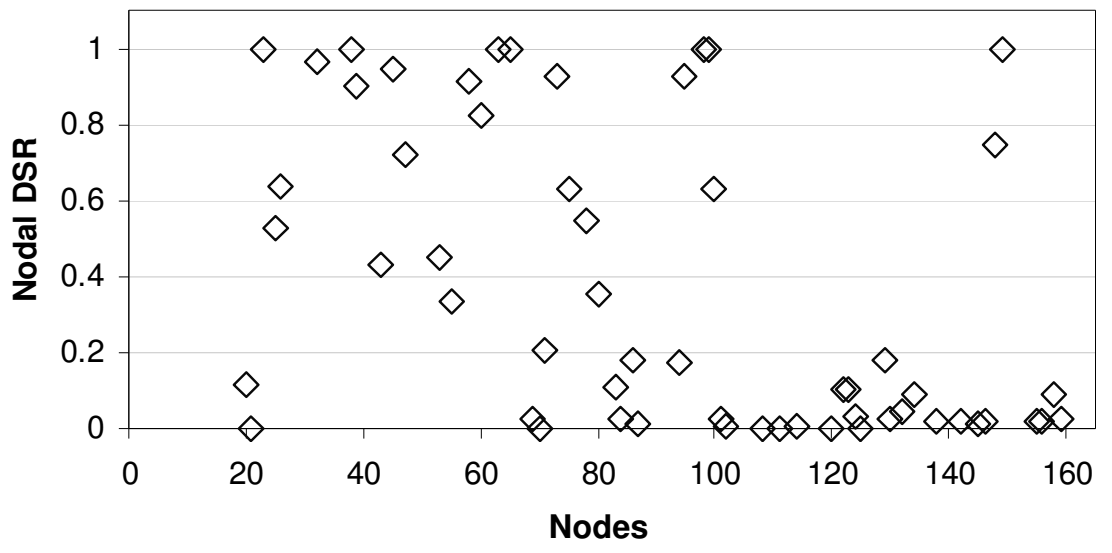

Fig. 12 Nodal DSRs for Network 4

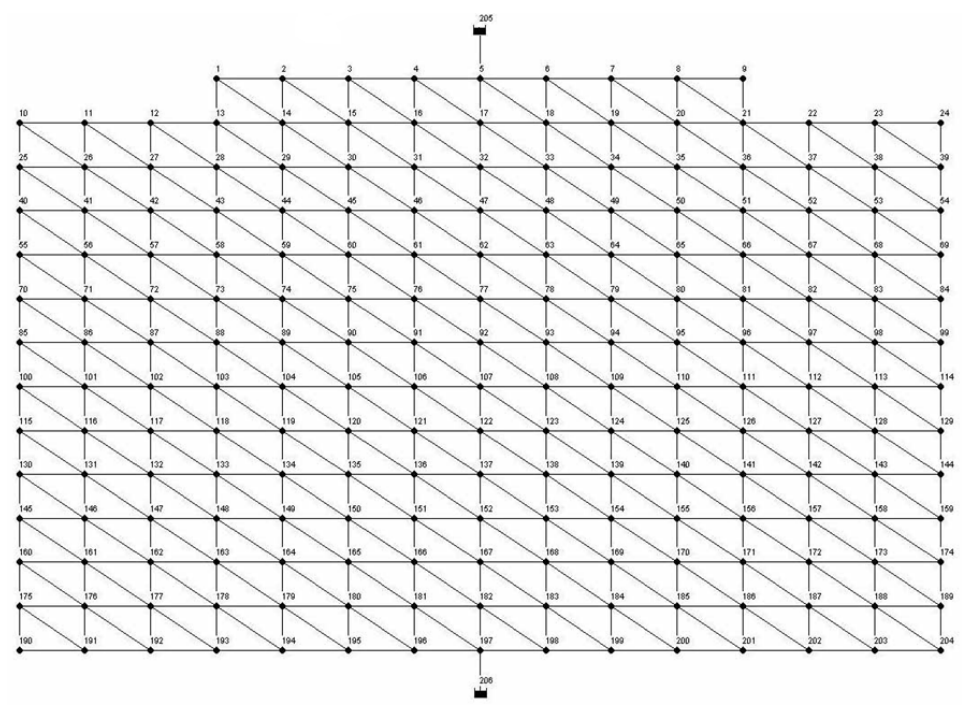

Fig. 13 Layout of Network 5 


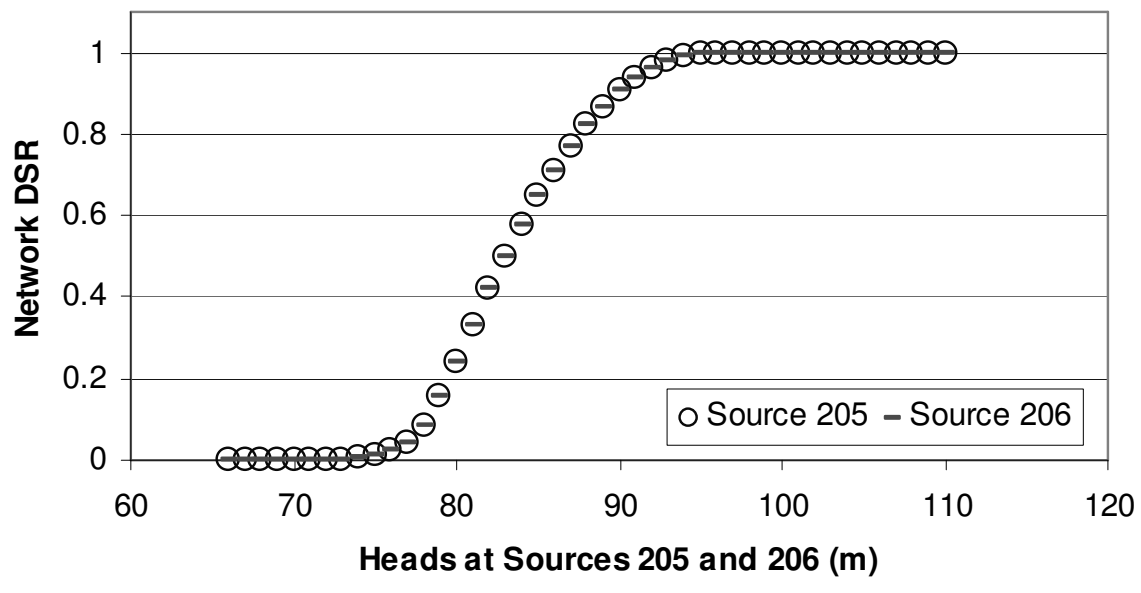

Fig. 14 Performance of Network 5

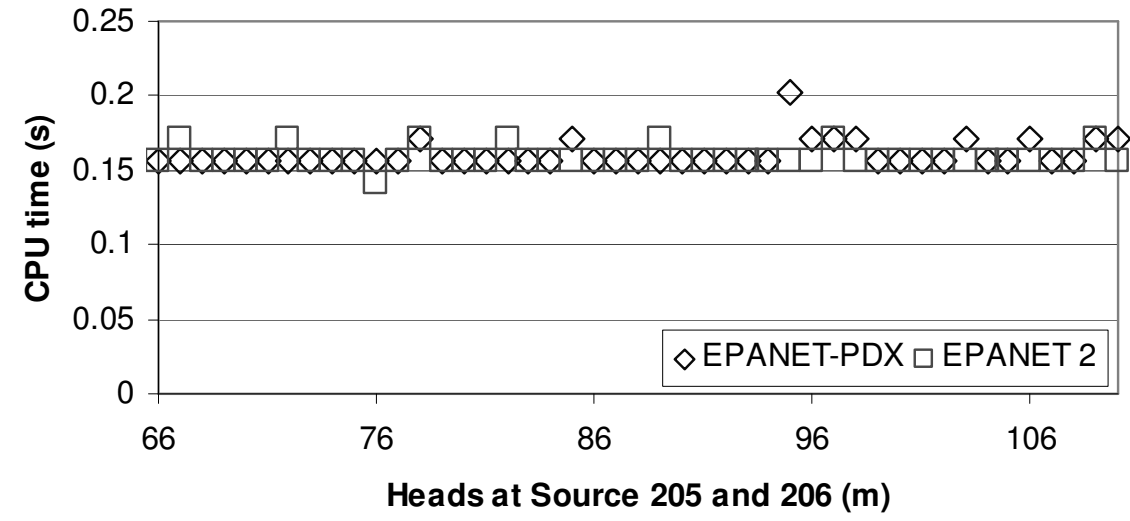

Fig. 15 Performance of EPANET-PDX and EPANET 2 for SHV simulations 


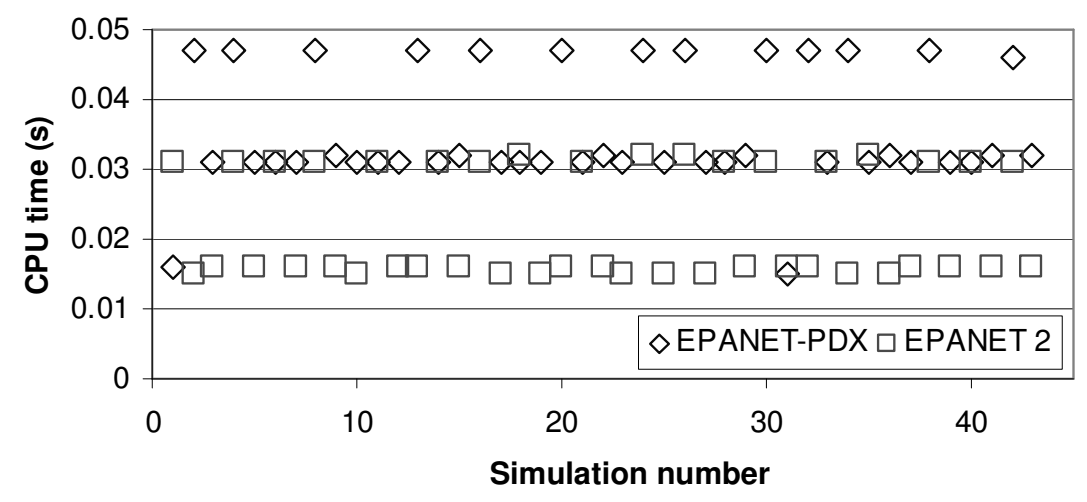

Fig. 16 Performance of EPANET-PDX and EPANET 2 for PC simulations

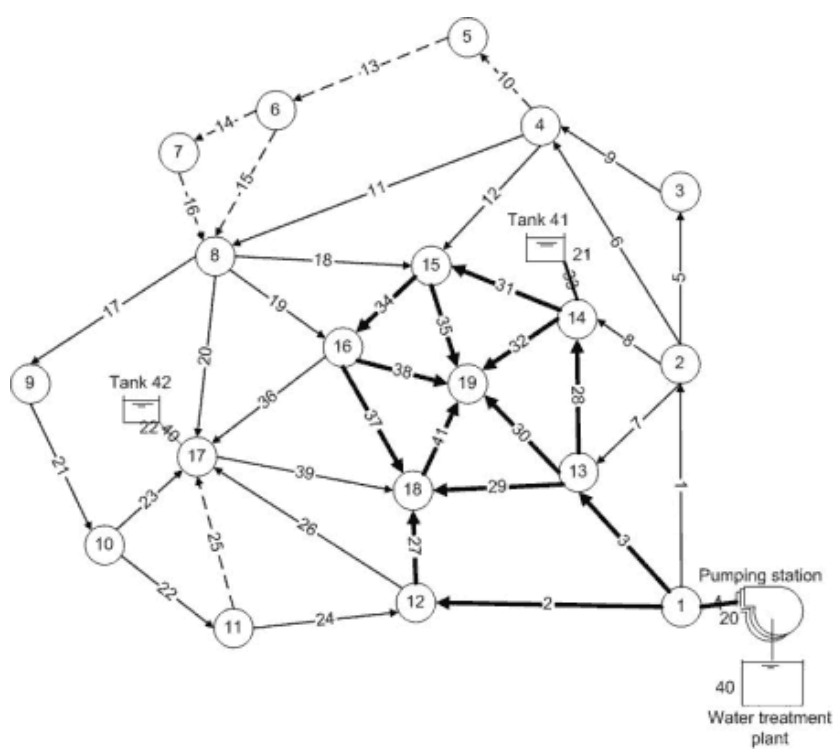

Fig. 17 Layout of Network 6 


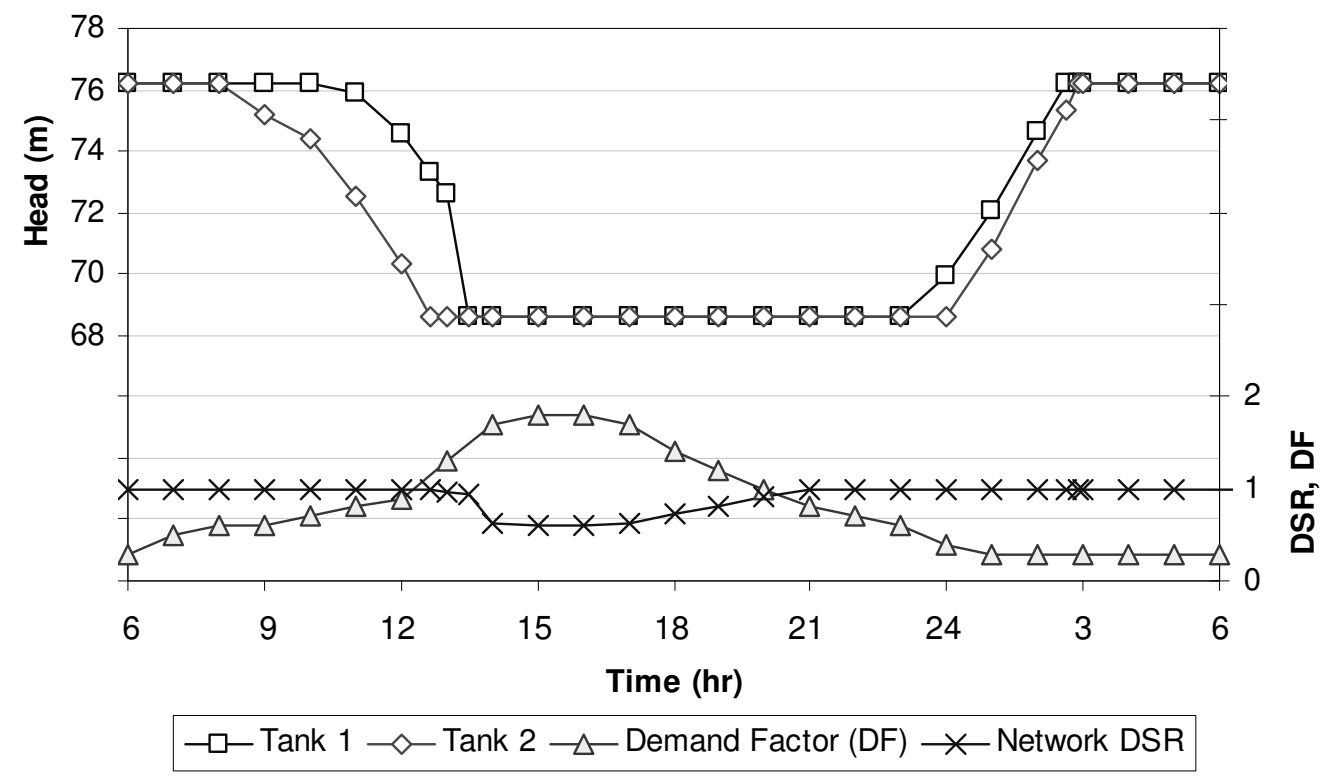

Fig. 18 Tank heads and network DSR results

Time 16:00 (Network DSR=0.6)

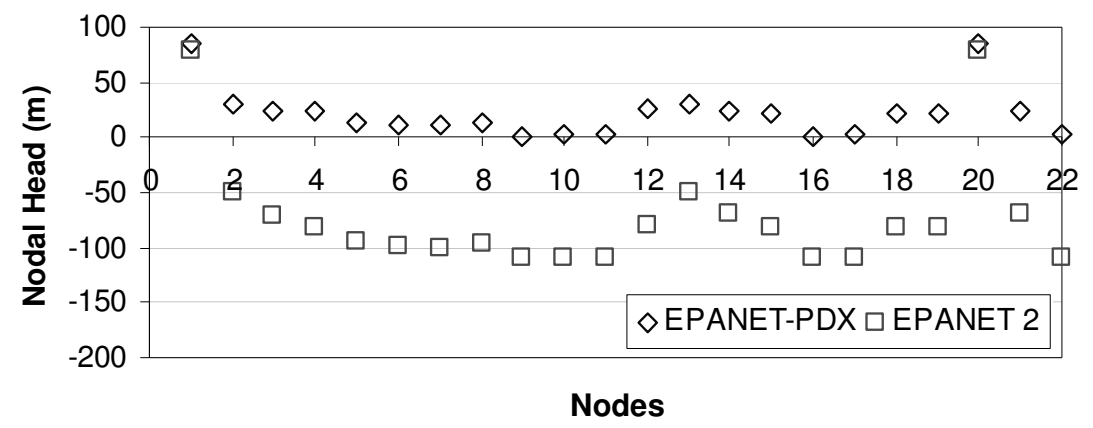

Fig. 19 Nodal heads for time 16:00 
Time 19:00 (Network DSR=0.8)

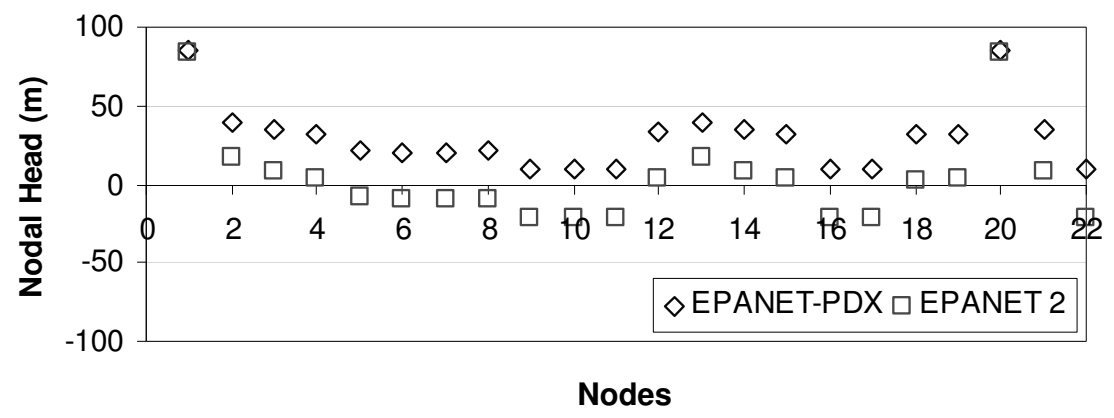

Fig. 20 Nodal heads for time 19:00

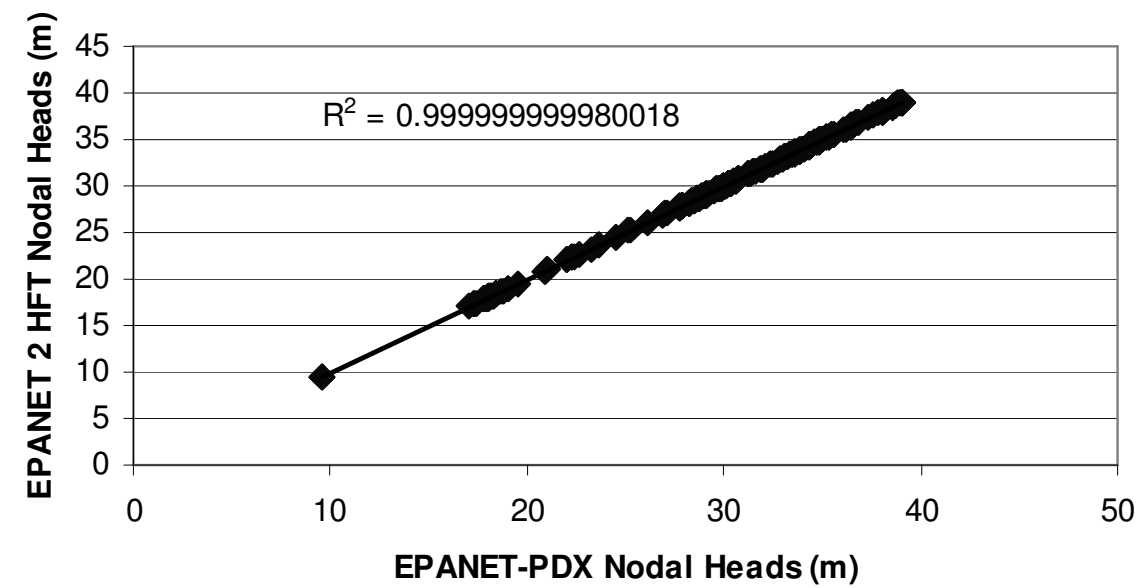

Fig. 21 Correlation between nodal heads of EPANET-PDX and EPANET 2 HFT (PC simulations for Network 2)

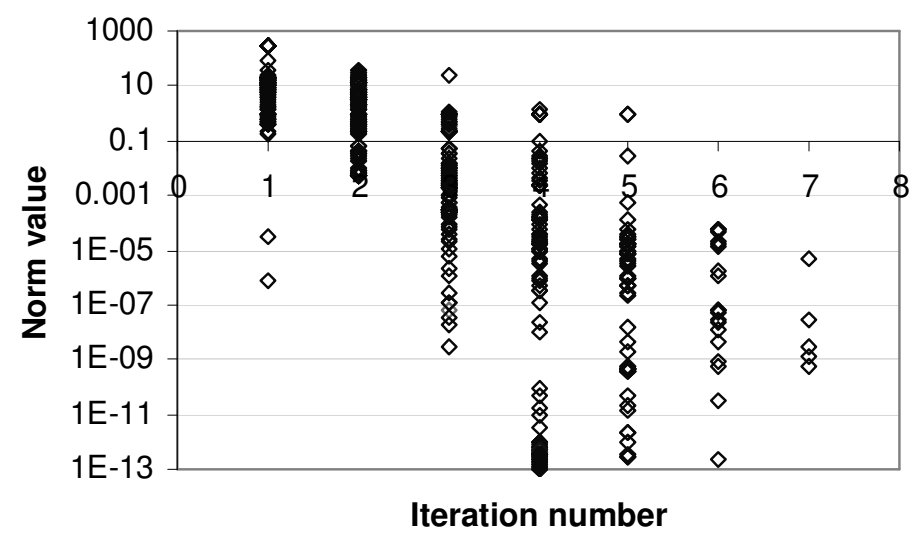

Fig. 22 Norm value for each iteration 


\section{Tables}

Table 1 Network details and number of simulations

\begin{tabular}{cccccc|cc}
\hline \multicolumn{5}{c|}{ Number of network elements indicated } & \multicolumn{2}{c}{ Number of simulations } \\
\hline $\begin{array}{c}\text { Network } \\
\text { number }\end{array}$ & Nodes & Pipes & Pumps & Valves & Sources & SHV & PC \\
\hline 1 & 6 & 8 & 0 & 0 & 1 & 48 & $7(1)$ \\
2 & 36 & 70 & 0 & 0 & 1 & 36 & $54(1-8)$ \\
3 & 9 & 9 & 2 & 1 & 2 & 72 & $15(1-3)$ \\
4 & 164 & 200 & 4 & 2 & 5 & 50 & $50(2-10)$ \\
5 & 204 & 557 & 0 & 0 & 2 & 45 & $43(5-10)$ \\
6 & 22 & 43 & 3 & 0 & 3 & $20^{*}$ & $10^{*}(2-5)$ \\
\hline
\end{tabular}

Values in parentheses ( ) represent the range of the number of pipes closed in the pipe closure simulations

* Extended Period Simulation

SHV - Source head variation simulations

PC - Pipe closure simulations

Table 2 Node data for Network 3

\begin{tabular}{ccccccccc}
\hline Node & 1 & 2 & 3 & 4 & 5 & 6 & $7-8$ & 9 \\
\hline Elevation $(\mathrm{m})$ & 150 & 150 & 100 & 130 & 130 & 150 & 120 & 120 \\
Demand $(1 / \mathrm{s})$ & - & 30 & - & 80 & 50 & 30 & - & 80 \\
\hline
\end{tabular}

Table 3 Performance of simulators for source head variation simulations

\begin{tabular}{|c|c|c|c|c|c|}
\hline \multirow{3}{*}{$\begin{array}{c}\text { Network } \\
1\end{array}$} & \multicolumn{2}{|c|}{ Average number of iterations } & \multicolumn{3}{|c|}{ Average CPU time (s) } \\
\hline & EPANET-PDX & EPANET 2 & \multicolumn{2}{|c|}{ EPANET-PDX } & EPANET 2 \\
\hline & (4) & (4) & 0.0478 & $(0.046)$ & $0.0489 \quad(0.046)$ \\
\hline 2 & $4.3056(4)$ & 4.3056 & 0.0640 & $(0.062)$ & $0.0673 \quad(0.063)$ \\
\hline 3 & $5.5753(5)$ & $5.5616 \quad(5)$ & 0.0585 & $(0.047)$ & $0.0543 \quad(0.047)$ \\
\hline 4 & $5.52 \quad(5)$ & 5.52 & 0.1697 & $(0.164)$ & $0.1666(0.164)$ \\
\hline 5 & $5.2889(5)$ & 5.2889 (5) & 0.157 & $(0.156)$ & $0.158 \quad(0.156)$ \\
\hline 6 & $4.102(4.111)$ & $3.171(3.148)$ & 0.0461 & $(0.046)$ & $0.0332(0.031)$ \\
\hline
\end{tabular}


Table 4 Performance of simulators for pipe closure simulations

\begin{tabular}{c|cc|cc}
\hline \multirow{2}{*}{ Network } & \multicolumn{2}{|c|}{ Average number of iterations } & \multicolumn{2}{c}{ Average CPU time (s) } \\
\cline { 2 - 5 } & EPANET-PDX & EPANET 2 & EPANET-PDX & EPANET 2 \\
\hline 1 & $5.4286(6)$ & $4.1428(5)$ & $0.0539(0.047)$ & $0.0419(0.046)$ \\
2 & $4.38(4)$ & $4.20(4)$ & $0.0691(0.062)$ & $0.0582(0.047)$ \\
3 & $5.0556(4)$ & $5.0556(4)$ & $0.0552(0.047)$ & $0.0524(0.047)$ \\
4 & $5.96(6)$ & $5.94(6)$ & $0.173(0.125)$ & $0.139(0.109)$ \\
5 & $4.860(5)$ & $5.837(6)$ & $0.0353(0.031)$ & $0.0222(0.016)$ \\
6 & $4.260(4.226)$ & $2.792(2.695)$ & $0.0484(0.047)$ & $0.0328(0.031)$ \\
\hline
\end{tabular}

Values in parentheses ( ) represent the median

Table 5 Correlation between nodal heads of EPANET-PDX and EPANET 2 HFT

\begin{tabular}{c|cc|cc}
\hline \multirow{2}{*}{ Network } & \multicolumn{2}{|c|}{ Source Head Variation } & \multicolumn{2}{c}{ Pipe Closure } \\
\cline { 2 - 5 } & $\begin{array}{c}\text { No. of } \\
\text { simulations } \\
\text { sampled }\end{array}$ & $1-R^{2}$ & $\begin{array}{c}\text { No. of } \\
\text { simulations } \\
\text { sampled }\end{array}$ & $1-R^{2}$ \\
\hline 1 & 22 & $1.56625 \times 10^{-5}$ & 7 & $5.2537 \times 10^{-11}$ \\
2 & 18 & $1.1389 \times 10^{-10}$ & 9 & $1.9982 \times 10^{-11}$ \\
3 & 15 & $5.801 \times 10^{-12}$ & 9 & $1.6335 \times 10^{-11}$ \\
4 & 11 & $2.6176 \times 10^{-6}$ & 10 & $8.4723 \times 10^{-8}$ \\
5 & 10 & $1.3121 \times 10^{-9}$ & 8 & $2.2984 \times 10^{-6}$ \\
6 & $5^{*}$ & $2.0624 \times 10^{-9}$ & $5^{*}$ & $1.2656 \times 10^{-10}$ \\
\hline
\end{tabular}

* Extended Period Simulation 
Table 6 Norm value at the last iteration of the simulation

\begin{tabular}{ccc}
\hline Network & Maximum value $(\mathrm{cfs}$ and $\mathrm{ft})$ & Mean value $(\mathrm{cfs}$ and $\mathrm{ft})$ \\
\hline 1 & $2.72550 \times 10^{-9}$ & $1.28297 \times 10^{-9}$ \\
2 & $3.64349 \times 10^{-5}$ & $2.30958 \times 10^{-6}$ \\
3 & $3.34541 \times 10^{-5}$ & $6.29189 \times 10^{-6}$ \\
4 & $6.32202 \times 10^{-5}$ & $2.73626 \times 10^{-5}$ \\
5 & $4.94513 \times 10^{-6}$ & $3.85161 \times 10^{-7}$ \\
6 & $4.20527 \times 10^{-6}$ & $3.9964 \times 10^{-5}$ \\
\hline
\end{tabular}

Values presented here are based on imperial units, i.e. cfs and ft for mass balance and energy balance respectively. $1 \mathrm{cfs}=0.02832 \mathrm{~m}^{3} \mathrm{~s}^{-1}, 1 \mathrm{ft}=0.3048 \mathrm{~m}$ 